\title{
Metabolic syndrome and the use of antipsychotics
} Vaios Peritogiannis*, Sofia Tsouli, Spyros Zafiris, Dimitrios Pappas and Venetsanos Mavreas

\author{
Address: Department of Psychiatry, University Hospital of Ioannina, Greece
}

* Corresponding author

from International Society on Brain and Behaviour: 2nd International Congress on Brain and Behaviour Thessaloniki, Greece. 17-20 November 2005

Published: 28 February 2006

Annals of General Psychiatry 2006, 5(SuppI I):S269 doi:I0.I I86/I744-859X-5-SI-S269

\begin{abstract}
Background
The introduction of second generation antipsychotics provided mental health professionals effective first line agents for the treatment of schizophrenia and other psychoses, which cause less side effects than first generation ones. The expanding use of these drugs is strongly correlated with the development of metabolic syndrome in the long-term, resulting in serious medical comorbidities of psychotic patients, such as cardiovascular events.
\end{abstract}

\section{Materials and methods}

A Medline search was conducted in order to retrieve papers concerning metabolic syndrome as a result of antipsychotic treatment. The key words were antipsychotics, schizophrenia, diabetes, hyperlipidemia, hypertriglyceridemia, metabolic syndrome. A total of 110 papers was revealed. 35 of them were used for the purpose of this study.

\section{Results}

Second generation antipsychotics are more likely than first generation ones to cause metabolic syndrome. The possible mechanisms are weight gain, insulin resistance, or a combination of these. It is possible that drugs with increased histamine $\mathrm{H} 1$ receptor affinity are more likely to cause metabolic syndrome. There are differences among them with this respect. Olanzapine is the compound associated with the greater incidence of weight gain, abnormalities in glucose-insulin homeostasis and lipid metabolism, thus resulting in the development of metabolic syndrome. Risperidone and quetiapine are less likely to cause this side effect, while ziprasidone appears having no impact in the development of metabolic syndrome. There are no sufficient data for amisulpride and aripiprazole. Clozapine has been strongly associated with meta- bolic adverse events, but is the most effective compound for the treatment of refractory schizophrenia.

\section{Discussion}

The choice of a second generation agent may be influenced by the potential risk for the development of metabolic syndrome. Some agents should be avoided or administrated with caution in patients being already at risk, such as diabetic ones, or patients with high lipid serum levels or a family history of metabolic abnormalities. If such adverse effects appear during treatment, dose reduction or switching to another agent may be appropriate. Weight, lipid and glucose serum levels should be monitored in patients receiving second generation antipsychotics and nutritional instructions should be given, as well as advice for physical exercise. Clozapine remains a special case as it is the most effective compound, but also the most likely to cause metabolic abnormalities. Careful management is required for patients who are at risk for metabolic syndrome while receiving clozapine.

\section{References}

I. Melkersson K, Dahl ML: Adverse metabolic effects associated with atypical antipsychotics: literature review and clinical implications. Drugs 2004, 64:701-723.

2. Nasrallah HA, Newcomer JW: Atypical antipsychotics and metabolic dysregulation: evaluating the risk/benefit equation and improving the standard of care. J Clin Psychopharmacol 2004, 25(suppl I):7-14.

3. Lambert BL, Chang KY, Tafesse E, Carson W: Association between antipsychotic treatment and hyperlipidemia among California medicaid patients with schizophrenia. J Clin Psychopharmacol 2005, 25: I2-18.

4. Gupta S, Stenmeyer C, Frank B, Madhusoodanan, Lockwood K, Lentz $B$, Keller P: Hyperglycemia and hypertriglyceridemia in real world patients on antipsychotic therapy. Am J Ther 2003, 10:348-355. 